\title{
Discussion on the limit cycles of the Lev Ginzburg equation by M. Bellamy and R.E. Mickens, Journal of Sound and Vibration, Vol. 308 (2007), 337-342
}

Claudio Aguinaldo Buzzi, Rodrigo Donizete Euzébio

Departamento de Matemática, IBILCE, UNESP,

Rua Cristóvão Colombo, 2265, Jardim Nazareth, CEP 15.054-000, São José do Rio Preto, SP, Brazil.

e-mail:buzzi@ibilce.unesp.br,rodrigo.euzebio@sjrp.unesp.br

Jaume Llibre

Departament de Matemàtiques, Universitat Autònoma de Barcelona, 08193 Bellaterra, Barcelona, Catalonia, Spain.

e-mail: jllibre@mat.uab.cat

Luis Fernando Mello

Instituto de Ciências Exatas, Universidade Federal de Itajubá, Avenida BPS 1303, Pinheirinho, CEP 37.500-903, Itajubá, MG, Brazil.

e-mail: lfmelo@unifei.edu.br

Corresponding author, Tel: 00-55-35-36291217, Fax: 00-55-35-36291140

Key words: Lev Ginzburg differential equation, limit cycle.

2000 MSC: 34C23, 34C25, 34C29 


\section{Introduction}

In his studies on population dynamics in 1986 Ginzburg [1] worked with the following family of second order differential equations

$$
\frac{\mathrm{d}^{2} x}{\mathrm{~d} t^{2}}+\alpha\left(1-\beta_{1} \frac{\mathrm{d} x}{\mathrm{~d} t}\right) x=\left(1-\beta_{1} \frac{\mathrm{d} x}{\mathrm{~d} t}\right)\left(\gamma+\beta \frac{\mathrm{d} x}{\mathrm{~d} t}\right)
$$

depending on four parameters: $\alpha>0, \beta_{1}>0, \gamma>0$ and $\beta \in \mathbb{R}$. Recently Bellamy and Mickens [2] claimed that the Lev Ginzburg differential equation (1) can exhibit a limit cycle coming from a Hopf bifurcation. We will show that this differential equation has neither a Hopf bifurcation, nor limit cycles.

The second order differential equation (1) can be written as the following first order planar polynomial differential system

$$
\begin{aligned}
& x^{\prime}=\frac{\mathrm{d} x}{\mathrm{~d} t}=y, \\
& y^{\prime}=\frac{\mathrm{d} y}{\mathrm{~d} t}=\left(1-\beta_{1} y\right)(\gamma-\alpha x+\beta y),
\end{aligned}
$$

of degree 2 , simply called a quadratic system in what follows. We denote by $\mathcal{X}: \mathbb{R}^{2} \rightarrow \mathbb{R}^{2}$ the vector field associated with the differential system (2), that is

$$
\mathcal{X}(x, y)=\left(y,\left(1-\beta_{1} y\right)(\gamma-\alpha x+\beta y)\right) .
$$

The study of the existence of limit cycles in (1) goes back to the original work of Ginzburg who obtained some important results by numerical analysis. The differential system (2) presents only one equilibrium point $p=(\gamma / \alpha, 0)$ 
for all values of the parameters. The existence of limit cycles in system (2) was studied in [2] via Hopf bifurcation analysis, claiming that a limit cycle is born at the equilibrium point $p$ by a Hopf bifurcation when $\beta=0$. But the Hopf bifurcation analysis presented in [2] is not correct. In fact, the linearization $D \mathcal{X}(p)$ of $\mathcal{X}$ at $p$ when $\beta=0$ has eigenvalues $\lambda_{1,2}= \pm i \sqrt{\alpha}$. Then the equilibrium point $p$ is either a center or a weak focus (see [3] for more details), and the standard Hopf bifurcation analysis can only be applied when the equilibrium is a weak focus, but the equilibrium point $p$ when $\beta=0$ is a center. Thus the main results of this paper are the following.

Theorem 1. The Lev Ginzburg differential system (2) for $\beta=0$ has a center. Proof. Now consider $\beta=0$. By a translation, a linear change of variables and a rescaling of the independent variable $t$, system (2) can be written as

$$
\dot{u}=-v+\beta_{1} u v, \quad \dot{v}=u \text {. }
$$

It is easy to check that this differential system has the first integral

$$
H=H(u, v)=e^{\beta_{1}\left(2 u-v^{2} \beta_{1}\right)}\left(u \beta_{1}-1\right)^{2},
$$

because

$$
\mathcal{X} H=\frac{\partial H}{\partial u}\left(-v+\beta_{1} u v\right)+\frac{\partial H}{\partial v} u=0
$$

The value of the first integral $H$ at the equilibrium point located at the origin is $H(0,0)=1$, and near the origin $H(u, v)<1$. Since $H(u, v)=$ 
$H(u,-v)$, we obtain that the curves $H(u, v)=h$ are symmetric with respect to the $u$-axis if they intersect such an axis. Therefore, since the origin is a focus or a center, it follows that the curves $H(u, v)=h \lesssim 1$ are closed, and consequently the origin is a center.

Theorem 2. There are no periodic orbits in the Lev Ginzburg differential system (2) for $\beta \neq 0$.

Proof. For each $\beta_{1}>0$, system (2) possesses an invariant line

$$
\mathcal{L}_{\beta_{1}}=\left\{(x, y) \in \mathbb{R}^{2}: x \in \mathbb{R}, y=1 / \beta_{1}\right\}
$$

that separates the plane in two disjoint invariant unbounded sets

$$
\mathcal{A}_{\beta_{1}}=\left\{(x, y) \in \mathbb{R}^{2}: x \in \mathbb{R}, y>1 / \beta_{1}\right\}
$$

and

$$
\mathcal{B}_{\beta_{1}}=\left\{(x, y) \in \mathbb{R}^{2}: x \in \mathbb{R}, y<1 / \beta_{1}\right\}
$$

that is, $\mathbb{R}^{2}=\mathcal{A}_{\beta_{1}} \cup \mathcal{B}_{\beta_{1}} \cup \mathcal{L}_{\beta_{1}}$. Now consider the vector field $\mathcal{Y}: \mathcal{A}_{\beta_{1}} \cup \mathcal{B}_{\beta_{1}} \rightarrow \mathbb{R}^{2}$ given by

$$
\mathcal{Y}(x, y)=\frac{1}{1-\beta_{1} y} \mathcal{X}(x, y)
$$

where $\mathcal{X}$ is defined in (3). The divergence of $\mathcal{Y}$ is given by

$$
\operatorname{div} \mathcal{Y}(x, y)=\beta \neq 0 \text {. }
$$


From the Bendixson criterion (see Theorem 7.10 of [4], for instance) it follows that the vector field $\mathcal{X}$ does not have any periodic orbit in the region $\mathcal{A}_{\beta_{1}} \cup \mathcal{B}_{\beta_{1}}$. The theorem is proved.

\section{Conclusions}

This paper shows that the Lev Ginzburg differential system (2) cannot exhibit a Hopf bifurcation. See Theorem 1. Concerning the existence of a limit cycle, the main result of this article is summarized in Theorem 2 which says that there are no limit cycles in system (2).

\section{Acknowledgments}

The first author thanks CNPq, CAPES and FAPESP for the financial support. The second author is supported by FAPESP, grant 2010/180156. The third author is partially supported by the MICINN/FEDER grant MTM2008-03437, AGAUR grant 2009SGR-410 and ICREA Academia. The fourth author is partially supported by CNPq grant 304926/2009-4 and by FAPEMIG grant PPM-00204-11. All the authors except the second one are also supported by the joint project CAPES-MECD grant PHB-2009-0025PC.

\section{References}

[1] L.R. GinzBurg, The theory of population dynamics, Journal of Theoretical Biology 122 (1986), 335-399. 
[2] M. Bellamy And R.E. Mickens, Hopf bifurcation analysis of the Lev Ginzburg equation, Journal of Sound and Vibration 308 (2007), 337-342.

[3] N.N. Bautin, On the number of limit cycles which the variations of coefficients from an equilibrium state of the type focus or center, American Mathematical Society Translations 100 (1954), 1-19.

[4] F. Dumortier, J. Llibre And J.C. Artés, Qualitative theory of planar differential systems, Universitext, Springer-Verlag, New York, 2006. 